\title{
Fouling mechanism and control strategy of inorganic membrane
}

\author{
Peng $\mathrm{Li}^{1}$, Shoubin Zhang, ${ }^{1, *}$, Ying Lv ${ }^{1}$, Guoqiang $\mathrm{Ma}^{1}$ and Xinyi Zuo ${ }^{1}$ \\ ${ }^{1}$ School of Civil Engineering \& Architecture, University of Jinan, NO.336, Nanxinzhuang West Road., Jinan 250022, P.R.China
}

\begin{abstract}
Compared with the traditional treatment technologies, inorganic membrane technology is gradually becoming the mainstream of the treatment of oily and salty wastewater, but membrane fouling has become the bottleneck restricting the development of membrane technology. In order to solve this problem, the mechanism of membrane fouling and the control strategies of membrane fouling are introduced in this paper. The atomic layer deposition technology and the preparation of $\mathrm{TiO}_{2}$ nanowires (NWs) film by impregnation method were mainly introduced, aiming to provide a more reliable industrial research status in the field of membrane fouling control.
\end{abstract}

\section{Introduction}

In recent years, with the enhancement of people's awareness of environmental sanitation and the increase of the demand for clean water, oil and water separation has attracted more and more attention ${ }^{[1]}$. Oily wastewater is a common product in petroleum, natural gas, food, steel and other chemical industries. At the same time, oilly wastewater is also produced when it is leakaged into open water during the exploration of crude oil and transportation of petroleum products, which makes the treatment of oily wastewater an important topic in the field of water treatment ${ }^{[2-4]}$.

At present, we always select these methods to treat the oily wastewater, such as gravity, centrifuge, electrostatic precipitation, cyclone separator, flotation, demulsification, heat treatment, adsorption, membrane separation and so on. Among these techniques, membrane separation becomes the wonderful choice with its unique advantages. Compared with traditional methods, membrane separation technology can remove oil droplets in oil-water emulsion more effectively ${ }^{[5]}$. And it has many advantages, such as high selectivity, low energy consumption, simple operation, good reliability, low maintenance cost and small floor space, etc $^{[3]}$. The microfiltration have gradually become a potential technology for the treatment of oily wastewater due to the appearance of shortages of traditional process treatment ${ }^{[6]}$. However, membrane fouling is usually regarded as its "fatal weakness"[7] because of the existance of membrane fouling in membrane treatment ${ }^{[8]}$, which severely limits the promotion and use of membrane technology ${ }^{[2]}$. Despite so many deficiencies it is, the advantages of membrane separation technology are still outstanding ${ }^{[9]}$. Membrane technology still has a broad development prospect in the treatment of oily and salty wastewater ${ }^{[6]}$. Therefore, it is imperative to study the mechanism of membrane fouling ${ }^{[1]}$ and develop new membrane fouling control strategies ${ }^{[8]}$.

\section{Membrane fouling mechanism}

\subsection{Membrane fouling mechanism}

Membrane fouling results from the interaction between pollutants and membranes, and it is generally believed that deposition/adhesion processes are determined by short-term thermodynamic interactions between flux and membrane surface ${ }^{[10]}$. The blocking law describes dirt can exist in three state- dirt is located at the top of the orifice, dirt penetrates into the orifice, or a piece of dirt forms on the surface of the film ${ }^{[1]}$. Soluble microbial products (SMP) and natural organic matter (NOM) are the main pollutants that cause membrane fouling. The filter cake layer is the most important form of fouling in the whole membrane filtration process. The initial filter cake layer is formed in the sufficient accumulation of dirt deposited on the membrane surface (larger than the membrane hole). With the passage of time, due to the penetration resistance, the filter cake gradually thickens with the deposition of more dirt. With the formation of the filter cake layer, the mass transfer resistance of the permeation process inevitably increases, leading to a decrease in the permeation flux under constant transmembrane pressure (TMP) or an increase in the permeation flux under constant flux operating condition. Further studies showed that both the cake thickness and porosity could affect the filtration performance. It was found that there was a porosity gradient on the cake thickness, and the porosity near the film was low.

Three characteristic stages of membrane fouling ${ }^{[6]}$ are as follows: (1) droplet attachment and aggregation; (2) droplet deformation; (3)droplet coalescence. The deformability of oil can be observed microscopically, as well as by the optical coherence microscop (OCT), and oil emulsion can cause internal pore scaling even if it is two orders of magnitude larger than the pore. The direct 
observation through the membrane (DOTM) method shows that salt reduces not only the solubility of the surfactant, but also the interfacial tension and Zeta potential of the oil emulsion, thus increasing the coalescence effect and increasing the volume of droplet is easy to be cut off by cross-flow.

\subsection{Relevant studies}

Membrane filtration is a promising method to treat a large amount of oily wastewate. However, oil emulsion filter cake scaling (filter cake scaling is the main form of the whole filtration process) is still unclear. In order to fill this gap, Henry $\mathbf{J}$ et al. used DOTM technology to characterize the evolution of the characteristics of the oil emulsion layer on the hollow fiber (HF) membrane in real time.

Renjie $\mathrm{Li}$ et al. adopted the extended DerjaguinLandau-Verwey-Overbeek (XDLVO) theory and density functional theory (DFT) to study the effect of membrane surface roughness on the adhesion pollution of sodium alginate. The combination of XDLVO and DFT not only provides a molecular basis for the study of membrane fouling, but also provides a new approach for the study of membrane fouling.

\section{Membrane fouling control strategies}

\subsection{Atomic layer deposition (ALD) technique}

Ning $\mathrm{Li}$ et al. studied membrane fouling of polyvinylidene fluoride (PVDF) microfiltration membranes in membrane bioreactors $(\mathrm{MBRs})^{[8]}$. They believed that proteins and polysaccharides, as the main components of extracellular polymeric substance (EPS), were considered as the main membrane fouling factors of MBRs. When proteins and polysaccharides are adsorbed and accumulated on the membrane surface, the membrane pores tend to be blocked, which leads to the decrease of membrane flux and water treatment efficiency ${ }^{[11-13]}$. The increase of membrane cleaning frequency increases the operating cost of MBRs by $40 \%$. Furthermore, the life of the membrane is shortened with frequent cleaning. Based on the above problems, it is urgent to develop effective membrane fouling control strategies to achieve efficient operation of MBRs, and hydrophilic modification is considered as an effective way to reduce membrane fouling.

In recent decades, various methods of membrane modification have been studied, including surface coating, immersion precipitation, chemical grafting and blending. Hydrophilic materials can be coated on the membrane surface or embedded into the membrane material by these methods, so as to change the physical and chemical properties of the membrane surface and bring better anti-pollution performance. However, these traditional methods still have some shortcomings in the transformation process. The surface coating is physical adsorption, whose coating is unstable, and it will lead to hydrophilic loss through the modified layer desorption. In addition, the grafting method can achieve stable coating by chemical bonds, but this modification process usually needs to be carried out in solution, and the most important is that we can hardly control this process. With the continuous reaction between the modified precursors, the long chain is more likely to grow in the membrane pore, which leads to the blockage of the membrane pore and the decrease of the permeation flux. In addition, the heterogeneous mixture of the modified material and the membrane material also appeared in the process of blending modification. Atomic layer deposition technology combines the advantages of self-limiting growth and atomic scale layer by layer deposition, and realizes uniform controllable modification of membrane surface and pore wall, which has become an advanced technology for membrane modification.

The sediment layer is closely bound to the membrane surface through chemical adsorption and chemical reactions.In addition, we can precisely control the thickness of the modified layer by adjusting the number of deposition cycles, which can effectively avoid pore clogging. In recent years, scholars have found that during ALD, the binding strength between membrane materials and different modified substances has changed, which will have a significant impact on the deposition rate of the modified layer. In addition, the growth rate of modified membrane is closely related to membrane flux and retention rate. Different modified materials have different effects on the physical and chemical properties of the membrane, showing great difference in antifouling performance. Therefore, we focused on the selection of hydrophilic materials with strong anti-pollution properties, and modified PVDF membrane by ALD technology.

Ning $\mathrm{Li}$ et al. used $\mathrm{TiO}_{2}, \mathrm{Al}_{2} \mathrm{O}_{3}, \mathrm{ZnO}$ and other common metal oxides as modified materials to modify PVDF membrane, and used bovine serum albumin (BSA) and sodium alginate (SA) as model sources of proteins and polysaccharides for antifouling studies. By comparing the three kinds of modified membrane, the best modified membrane was selected.The adsorption isotherm and thermodynamics were used to explain the scale prevention mechanism ${ }^{[14]}$. At the same time, static adsorption and filtration experiments showed that $\mathrm{ZnO}$ modified membrane had the lowest adsorption capacity for BSA and SA, the lowest decrease in pure water flux, and the lowest increase in resistance after membrane fouling. In addition, adsorption isotherm analysis showed that there was monolayer adsorption on the modified $\mathrm{ZnO}$ film. Adsorption of BSA and SA on the $\mathrm{ZnO}$ layer obtained the lowest thermodynamic parameter values of $\Delta \mathrm{H}$ and $\Delta \mathrm{S}$, which shows that the relatively homogeneous distribution of impurities on the membrane surface and the weakest interaction between impurities and $\mathrm{ZnO}$ modified membrane. The adsorption of BSA by $\mathrm{ZnO}$ modified membrane was -3.92 $3.21 \mathrm{kj} / \mathrm{mol}$, and the adsorption of $\mathrm{SA}$ was -0.95 $0.43 \mathrm{kj} / \mathrm{mol}$, which meant that the spontaneous probability of dirt adsorption decreased. In addition, AFM observation and water contact angle measurement showed that $\mathrm{ZnO}$ modified film had smooth surface and enhanced hydrophilicity. Based on the above analysis, the uniformly distributed $\mathrm{ZnO}$ modified film was 
considered as the best modified film to be screened.Thermodynamic parameters of BSA and SA adsorption on different films are shown in Table 1 (a), (b).

Table 1 (a). Thermodynamic parameters of BSA adsorption on different films.

\begin{tabular}{|c|c|c|c|c|c|}
\hline \multirow{2}{*}{ Parameters } & \multirow{2}{*}{$\begin{array}{c}\text { Temperature } \\
(\mathrm{K})\end{array}$} & \multicolumn{5}{|c|}{ BSA } \\
\cline { 3 - 6 } & & PVDF & PVDF-TiO $_{2}$ & PVDF-Al $_{2} \mathrm{O}_{3}$ & PVDF-ZnO \\
\hline$\Delta \mathrm{H}(\mathrm{kJ} / \mathrm{mol})$ & & 37.72 & 29.45 & 30.98 & 6.98 \\
\hline$\Delta \mathrm{S}(\mathrm{J} / \mathrm{mol} / \mathrm{K})$ & & 174.64 & 143.34 & 149.44 & 35.33 \\
\hline$\Delta \mathrm{G}(\mathrm{kJ} / \mathrm{mol})$ & 288 & -12.52 & -11.70 & -11.91 & -3.21 \\
\hline & 298 & -14.46 & -13.56 & -13.87 & -3.50 \\
\hline & 308 & -16.00 & -14.55 & -14.88 & -3.92 \\
\hline
\end{tabular}

Table 1（b). Thermodynamic parameters of SA adsorption on different films.

\begin{tabular}{|c|c|c|c|c|c|}
\hline \multirow{2}{*}{ Parameters } & \multirow{2}{*}{$\begin{array}{c}\text { Temperature } \\
(\mathrm{K})\end{array}$} & \multicolumn{4}{|c|}{$\mathrm{SA}$} \\
\cline { 3 - 6 } & & $\mathrm{PVDF}$ & $\mathrm{PVDF}-T i O_{2}$ & $\mathrm{PVDF}^{-\mathrm{Al}_{2} \mathrm{O}_{3}}$ & PVDF-ZnO $^{-}$ \\
\cline { 3 - 6 }$\Delta \mathrm{H}(\mathrm{kJ} / \mathrm{mol})$ & & 30.69 & 7.14 & 14.29 & 7.14 \\
\hline$\Delta \mathrm{S}(\mathrm{J} / \mathrm{mol} / \mathrm{K})$ & & 117.29 & 28.41 & 51.04 & 26.16 \\
\hline$\Delta \mathrm{G}(\mathrm{kJ} / \mathrm{mol})$ & 288 & -3.12 & -1.07 & -0.57 & -0.43 \\
\hline & 298 & -4.18 & -1.27 & -0.60 & -0.59 \\
\hline & 308 & -5.47 & -2.17 & -1.61 & -0.95 \\
\hline
\end{tabular}

\subsection{Preparation of $\mathrm{TiO}_{2}$ nanowires $\left(\mathrm{TiO}_{2} \mathrm{NWs}\right)$ membrane}

Using $\mathrm{TiO}_{2}$ nanoparticles as raw material, Zihe Pan et al. synthesized $\mathrm{TiO}_{2} \mathrm{NWs}$ through hydrothermal reaction and successfully coated it on various stainless steel films ${ }^{[4]}$ to improve its surface wettability (from super hydrophilic to super hydrophobic). The effect of loading $\mathrm{TiO}_{2} \mathrm{NWs}$ on hydrophobicity and oil-water separation efficiency is discussed by them ${ }^{[15]}$. In addition, they demonstrated a synergistic effect of wettability and pore size on oil-water separation. When the contact angle of water increases from $0^{\circ}$ to about $50^{\circ}$, the flow decreases with the increase of the contact angle of water, while the separation efficiency remains relatively stable. When the contact angle of water is between $90^{\circ}$ and $100^{\circ}$, the film loses the oil-water separation efficiency ${ }^{[16]}$. When the contact angle of water continues to increase to more than $120^{\circ}$, the prepared film changes into oil-removing type ${ }^{[17]}$, which contributes to the oil can pass through the film while maintains a hydrophobe state. Pore diameter also plays an important role in oil-water separation. The synergistic effect of wettability and pore diameter shows that under a certain pore diameter, superhydrophilicity is more important to improve the oil-water separation effect. It has been proved by experiments that the membrane prepared by them still has high durability and excellent anti-pollution performance after long-term operation.

\section{Conclusion}

Inorganic microfiltration membrane has been widely used in wastewater containing oil and salt, but membrane fouling has become a bottleneck restricting its development. The advantages of membrane treatment have led to the emergence of a large number of treatment technology of membrane fouling, atomic layer deposition technology has become an advanced technology because of its controllable advantages in the field of membrane modification. Of course, $\mathrm{TiO}_{2}$ nanowire film prepared by impregnation method also has its unique advantages. In the future, there will be more and more in-depth studies on the explanation of membrane fouling mechanism, and the revelation of the mechanism will be accompanied by a large number of new technologies, which is the direction we need to work together.

\section{Acknowledgement}

This work was financially supported by the Shandong Provincial Key Research and Development Program (2017GSF17105).

\section{References}

1. H.J. Tanudjaja, J.W. Chew. In-situ characterization of cake layer fouling during crossflow microfiltration of oil-in-water emulsion[J]. Separ. Purif. Technol, 218, 51-58(2019)

2. M. Abdalla, M. Nasser, A. Kayvani Fard, et al. Impact of combined oil-in-water emulsions and particulate suspensions on ceramic membrane 
fouling and permeability recovery[J]. Separ. Purif. Technol, 212, 215-222(2019)

3. M. Tawalbeh, A. Al Mojjly, A. Al-Othman, et al. Membrane separation as a pre-treatment process for oily saline water[J]. Desalination 447, 182-202 (2018)

4. Z. Pan, S. Cao, J. Li, et al. Anti-fouling $\mathrm{TiO}_{2}$ nanowires membrane for oil/water separation: Synergetic effects of wettability and pore size[J]. J. Membr. Sci, 572, 596-606( 2019)

5. S. Basu, G. Ghosh, S. Saha. Adsorption characteristics of phosphoric acid induced activation of bio-carbon: Equilibrium, kinetics, thermodynamics and batch adsorber design[J]. Process Saf. Environ. Prot, 117, 125-142(2018)

6. H.J. Tanudjaja, J.W. Chew. Assessment of oil fouling by oil-membrane interaction energy analysis[J]. J. Membr. Sci, 560, 21-29( 2018)

7. H.J. Tanudjaja, V.V. Tarabara, A.G. Fane, et al. Effect of cross-flow velocity, oil concentration and salinity on the critical flux of an oil-in-water emulsion in microfiltration[J]. J. Membr. Sci, 530: 11-19(2017)

8. N. Li, Y. Tian, J. Zhao, et al. Static adsorption of protein-polysaccharide hybrids on hydrophilic modified membranes based on atomic layer deposition: Anti-fouling performance and mechanism insight[J]. J. Membr. Sci, 548, 470480(2018)

9. Z. He, S. Kasemset, et al. The effects of salt concentration and foulant surface charge on hydrocarbon fouling of a poly(vinylidene fluoride) microfiltration membrane[J]. Water Res, 117, 230241 (2017)

10. R. Li, Y. Lou, et al. Effects of surface morphology on alginate adhesion: Molecular insights into membrane fouling based on XDLVO and DFT analysis[J]. Chemosphere 233, 373-380 (2019)

11. M.I. Konggidinata, B. Chao, et al. Equilibrium, kinetic and thermodynamic studies for adsorption of BTEX onto Ordered Mesoporous Carbon (OMC)[J]. J. Hazard. Mater, 336,249-25 9 (2017)

12. R. Zhang, W. Shi, et al. Influence of salts, anion polyacrylamide and crude oil on nanofiltration membrane fouling during desalination process of polymer flooding produced water[J]. Desalination $373,27-37(2015)$

13. Z. Zhao, S. Shi, et al. Comparative studies on fouling of homogeneous anion exchange membranes by different structured organics in electrodialysis[J]. J. Environ. Sci, 77, 218-228, (2019)

14. D. Pintossi, M. Saakes, et al. Electrochemical impedance spectroscopy of a reverse electrodialysis stack: A new approach to monitoring fouling and cleaning[J]. J. Power Sources 444 , 1-8(2019)

15. A.M. Awad, R. Jalab, et al. Adsorption of organic pollutants by nanomaterial-based adsorbents: An overview[J]. J. Mol. Liq, 301,1-31(2020)
16. Y.W. Berkessa, Q. Lang, et al. Anion exchange membrane organic fouling and mitigation in salt valorization process from high salinity textile wastewater by bipolar membrane electrodialysis[J]. Desalination 465, 94-103(2019)

17. D. Gang, Z.U. Ahmad, et al. A review of adsorptive remediation of environmental pollutants from aqueous phase by ordered mesoporous carbon[J]. Chem. Eng. J, 403,1-20(2021) 